\title{
Politique
}

\section{Les contraintes dans les relations entre le Québec et les États-Unis}

\section{Bruno Perron}

Numéro 7, hiver 1985

Projection internationale du Québec

URI : https://id.erudit.org/iderudit/040476ar

DOI : https://doi.org/10.7202/040476ar

Aller au sommaire du numéro

Éditeur(s)

Société québécoise de science politique

ISSN

0711-608X (imprimé)

1918-6584 (numérique)

Découvrir la revue

Citer cet article

Perron, B. (1985). Les contraintes dans les relations entre le Québec et les États-Unis. Politique, (7), 9-31. https://doi.org/10.7202/040476ar d'utilisation que vous pouvez consulter en ligne.

https://apropos.erudit.org/fr/usagers/politique-dutilisation/ 


\title{
Les contraintes dans les relations entre le Québec et les États-Unis
}

\author{
Bruno Perron \\ University Consortium for Research on North America
}

D'entrée de jeu, l'analyse des relations internationales du Québec pose l'épineux problème de la méthode d'analyse. Sans s'attarder trop longuement sur la question, une mise au point apparaît indispensable.

Les relations internationales sont généralement appréhendées dans la perspective d'un État jouissant d'une souveraineté nationale, délimité géographiquement par des frontières intangibles et doté d'une économie nationale. Aucun de ces éléments n'a de valeur absolue ni au Québec, ni ailleurs. Le développement de la division internationale du travail, lié à l'expansion des rapports politiques bilatéraux et/ou multilatéraux, limite l'autonomie des dirigeants politiques dans les choix qu'ils posent. Or, le Québec ne constitue pas un «État achevé»; l'extension du champ d'autonomie des provinces est régie par la Constitution canadienne, et les dirigeants provinciaux doivent se plier aux règles du fédéralisme canadien. Le concept d'espace me semble plus apte à cerner le processus d'interrelations des entités politiques.

L'espace caractérise une réalité en situant les rapports de force multiples qui s'exercent entre les acteurs concernés. L'enjeu et les forces structurantes sont deux facettes de l'analyse de l'espace. 
Il comporte des instances économique, idéologique, sociale et politique. Leur contrôle est convoité par des groupes sociaux particuliers, soit de manière à en consolider l'épicentre, soit pour s'en distancer.

Cette démarche vise à dissiper l'illusion que le Québec peut engager des relations d'égal à égal avec des acteurs de la scène mondiale. Des influences à la fois structurelles et conjoncturelles s'imposent de tout leur poids aux décideurs du Québec, qui nous interdisent de nous limiter aux seules manifestations de ces relations.

Les divers processus entrant en interaction, doivent être cernés; il s'agit du mouvement de structuration économique, de la consolidation politique, de la socialisation et de l'acculturation. De ce point de vue, la politique intérieure d'un pays subit des pressions extérieures qui déterminent, ou conditionnent tout au moins, la prise de décision. Réciproquement, les relations extérieures d'un gouvernement traduiront les tensions économiques, politiques et sociales, propres à la formation en question et elles porteront hors frontière la réponse des responsables politiques aux paradoxes intérieurs et extérieurs. Cette action politique déterminante exprime de façon condensée l'existence d'un espace politique qui outrepasse le cadre de l'entité politique étudiée, qu'il s'agisse du Québec, du Canada, des États-Unis, ou d'ailleurs. Le même phénomène prévaut pour l'économie dont le développement fait intervenir des ressources humaines et techniques exogènes et endogènes. Or, à cette étape-ci du développement de l'infrastructure économique et de la parcellisation du processus productif, besoin est de concevoir les rapports économiques à partir d'une notion plus extensive que celle de l'État-nation et de l'économie nationale. Or, le concept d'espace semble compiler la double qualité de permettre la compréhension des rapports internes à une région, tout en intégrant les facteurs extérieurs.

La première partie du travail présentera les principaux éléments de l'espace politique dans lequel le Québec est intégré. Ensuite, 
l'espace économique québécois sera présenté en abordant les secteurs d'activités dominants et les facteurs de prédiction. Les interrelations de la formation québécoise et de la formation sociale américaine seront cernées pour enfin déboucher sur les paradoxes qui entourent les choix politiques au Québec. Ceux-ci tiennent principalement à l'articulation entre le politique et l'économique dont la nature est déterminée par les choix politiques des responsables gouvernementaux.

\section{A. L'espace politique}

\section{a) Les prérogatives fédérales}

La province de Québec faisant partie d'un système fédéral, ses relations extérieures sont soumises à une certaine censure du gouvernement d'Ottawa. La Constitution canadienne n'établit pas les responsabilités respectives des deux paliers de gouvernement en la matière, ce qui a permis aux gouvernements provinciaux, notamment au Québec, d'entreprendre des initiatives. Toutefois, celles-ci sont cautionnées par la volonté politique à Ottawa d'entériner ou non la démarche de Québec. Dans le cas des relations avec les États-Unis, le fédéral détient un instrument de poids pour empêcher l'institutionnalisation de l'axe Québec-Washington. Le gouvernement provincial se heurte à des limites d'ordre politique, même si constitutionnellement rien n'interdit à ses responsables d'entretenir des relations extérieures officielles.

\section{b) Les rapports stratégiques}

L'État québécois n'est pas davantage autonome au plan des rapports stratégiques mondiaux. De façon plus extensive, le Québec est partie prenante d'un espace politique nord-américain qui impose certaines données à ses parties. Ainsi, le Québec subit des contraintes qui découlent des prises de position dans le conflit est-ouest, 
notamment en matière de défense, de relations commerciales, de politiques étrangères et d'organisation économique.

Un développement rapide des relations avec un pays jugé hostile aux États-Unis pourrait être interprété comme de la provocation et de la subversion. Les États-Unis ont un intérêt stratégique à maintenir le Québec, comme toutes les autres régions de l'Occident, en conformité avec les attentes de Washington sur les grandes questions à l'ordre du jour. Les responsables de la vie politique américaine visent à atteindre une homogénéité maximale des déclarations de principes au sujet de la réalité internationale. Les attitudes nationalistes au Québec les agacent car ils préferent maintenir un Canada fort et uni plutôt que de transiger avec plusieurs gouvernements au nord de leur frontière. Cette position limite beaucoup la marge de manœuvre du Québec dans ses relations avec Washington.

\section{c) Les négociations multilatérales}

Les relations multilatérales influencent également l'évolution des relations bilatérales, comme celles entre le Québec et les ÉtatsUnis. Ces relations intègrent les grands débats de fond et les responsables des organisations concernées essaient de dégager un consensus autour des actions à poser pour contrer des problèmes communs. Ceci constitue un nouveau mode de régulation qui favorise une vision globale des rapports politiques, économiques et idéologiques depuis 1945; le GATT, le FMI, la Banque Mondiale en sont des illustrations concrètes. En tant que signataire du GATT, membre de l'OCDE et des grandes organisations internationales, le Canada contracte des engagements internationaux. Ces obligations prises par le Canada limitent également l'action extérieure du Québec, tout en imposant certaines normes, principalement en matière d'investissement international et de commerce extérieur. L'espace politique dans lequel est inséré le Québec 
s'étend au-delà des frontières de la province ou du pays, ce qui n'implique nullement une surdétermination étrangère. Néanmoins, ces variables constitutionnelles, stratégiques et multilatérales contribuent aux tensions que rencontrent les décideurs québécois, responsables de la formulation d'une politique extérieure. Elles vont souvent à l'encontre de la structuration de cette politique, mais elles sont aussi des éléments complémentaires de l'État québécois sous sa forme actuelle. L'articulation entre ses politiques est médiatisée par ces variables.

\section{B. L'espace économique}

a) L'évolution industrielle

Ce qui a été dit de l'espace politique pourrait être repris pour l'espace économique. Car y a-t-il une économie nationale? La nature des interactions économiques entre le Québec et l'extérieur vont au-delà d'un flux de marchandises. Des liens structuraux unissent l'espace économique québécois à l'espace américain depuis que les États-Unis se sont tournés vers le Québec au début du siècle, pour satisfaire leurs besoins de pâte à papier, de cuivre, de fer et d'autres richesses naturelles. Un mode de développement continentaliste s'est instauré qui incorpore l'évolution économique du Québec aux besoins des centres industriels américains constitués ${ }^{1}$.

Le développement économique de la province a relevé des facteurs économiques, technologiques et géographiques dont le contrôle est souvent localisé aux États-Unis. Les produits forestiers, les ressources minières, l'énergie hydro-électrique et la maind'œuvre bon marché ont été des éléments fondamentaux de notre 1. C. Nappi, La structure des exportations du Québec. Montréal, C. D. Howe,
1978 , p. 9. 1. C. Nappi, La structure des exportations du Québec. Montréal, C. D. Howe,
1978 , p. 9. 
croissance économique ${ }^{2}$. Et celle-ci a emprunté la voie de l'internationalisation. L'exploitation des matières premières était utilisée comme élément catalyseur du développement industriel. Aujourd'hui, cette situation n'a guère changé car malgré la présence d'entreprises dynamiques, les ressources naturelles constituent toujours le pilier de notre développement économique.

Structurellement, nos échanges économiques dépendent des marchés américains pour les flux d'investissements, de technlogie et de marchandises. Par le fait même, toute fluctuation conjoncturelle aux États-Unis a des retombées importantes sur la dynamique des activités économiques de la province. Tel qu'il est mentionné précédemment, il ne faut pas confondre les manifestations et le processus dont elles découlent. Ce processus d'industrialisation a été amorcé sous le signe de l'internationalisme et de l'investissement étranger.

\section{b) Les secteurs d'activités}

Le recours au capital étranger n'est pas un phénomène récent puisqu'il remonte à l'origine même de la colonie. Depuis la $1^{\text {ère }}$ guerre mondiale, les Américains ont supplanté les Britanniques en tant que partenaire économique du Canada. Ce renversement d'alliance correspondit à une nouvelle forme d'investissement; l'investissement direct a remplacé l'investissement de portefeuille depuis les années cinquante. En terme concret, la firme est devenue le moyen d'augmenter la croissance économique et d'accroître la part des échanges mondiaux. Les spécificités de l'expérience québécoise se dégagent à la lumière du développement de ses secteurs

2. G. Gaudet, «Forces Underlying the Evolution of Natural Resource Policies in Quebec", dans Natural Resources in U.S.-Canadian Relations, vol. 1, édité par C. E. Beigie et A. O. Hero. Boulder, Westview Press, 1980, p. 248. 
d'activité. Ce recours au capital étranger ne signifie pas seulement l'acquisition d'une nouvelle technologie; il entraîne aussi des méthodes de gestion, de même que l'intégration à des réseaux d'échange contrôlés par la firme ${ }^{3}$.

La structure économique devient un complément de celle du géant américain qui se traduit par une dépendance structurelle. Le tissu industriel provincial évolue dans un cadre quasi dualiste. On retrouve d'une part les petites et moyennes entreprises autochtones, avec quelques exceptions, notamment Bombardier, dirigées par une bourgeoisie locale, et d'autre part, les grandes firmes multinationales (FMN) ${ }^{4}$. La diffusion de technologie se fait inégalement, les grandes entreprises œuvrant de façon exclusive dans leurs propres circuits. Elles contrôlent la propriété industrielle dans certains secteurs, nommément dans l'industrie du pétrole et du charbon, celles du tabac et de la machinerie, l'industrie chimique, celles des principaux métaux et des minéraux non métalliques, les industries d'instruments de précision, du caoutchouc, des appareils électriques, du matériel de transport, et autres ${ }^{5}$. Les industries manufacturières sont sous le contrôle étranger dans une proportion de 40 à $45 \%$ et le contrôle est total dans le secteur pétrolier, la houille, le caoutchouc et le tabac. Il est également très élevé dans le secteur minier, $80 \%$ des revenus imposables étant sous le contrôle du capital étranger ${ }^{6}$. En 1979, la contribution du capital étranger en général sur l'emploi se chiffrait aux alentours

3. B. Bonin, «U.S.A. - Quebec Economic Relations: Some Interactions Between Trade and Investment", dans Problems and Opportunities in U.S. - Quebec Relations, A. O. Hero et M. Daneau (eds.), Boulder, Westview Press, 1984, p. 25.

4. Cette dichotomie a été développée entre autres par: M. Saint-Germain, Une économie à libérer, Montréal, PUM, 1973.

5. B. Bonin, "U.S.A. - Quebec Economic Relations: Some interactions between Trade and Investment, " op. cit., p. 31.

6. B. Bonin, "U.S.A. - Quebec Economic Relations: A. Background Paper," Les Cabiers du Cetai, no. 82-01, Montréal, HEC, janvier 82, p. 5. 
de $35 \%^{7}$. De plus, il est à noter que les FMN contrôlent $40 \%$ des exportations de la province ${ }^{8}$.

Les activités liées à l'exploitation des richesses naturelles recueillent une partie importante des mises de fonds étrangères. En contrepartie, les entreprises nationales sont mal intégrées aux réseaux des firmes multinationales. Celles à propriété canadienneanglaise dominent les industries de l'édition, du bois en grume, du bois de pulpe à papier, de même que l'industrie du meuble. De leur côté, les Canadiens-français sont présents surtout dans les industries de produits métalliques, dans l'agro-alimentaire et dans le domaine des transports?.

La théorie économique néo-libérale postule que la présence des FMN procure des avantages en matière de technologie et de mise en marché, d'économies d'échelle et de revenus. En d'autres termes, la firme est perçue comme un instrument de maximisation de l'efficacité et du bien-être économiques ${ }^{10}$, à partir de capitaux bon marché.

Au Québec, l'implantation massive des FMN correspondit à une époque où les taux de croissance économique étaient élevés, la modernisation du tissu industriel accélérée, l'augmentation de tous les revenus rapide, de même que le niveau de vie amélioré. En dépit du fait que la FMN n'a pas été l'unique agent économique à participer à cet effort de récupération, son apport aura été

7. Statistique Canada, Industries manufacturières du Canada: niveau infraprovincial. Ottawa, Imprimeur de la Reine, 1979, pp. 7-11.

Le Groupe de recherche sur les élites industrielles au Québec, Rapport préliminaire présenté au ministère de l'Industrie et Commerce du Québec. Janvier 1976, p. 130.

8. Secrétariat au Développement Économique du Québec. Aperçu de la situation économique du Québec en 1982. Québec, l'Éditeur Officiel, 1982, p. I.

9. B. Bonin, «U.S.A. - Quebec Economic Relations: Some interactions..., " op. cit., p. 31.

10. N. Hood et S. Young, The Economics of Multinational Enterprise. Lan et $\mathrm{Ny}$. Longman, 1979, p. 179-325. 
substantiel. Toutefois, le développement global de l'économie s'est effectué à la faveur de certains secteurs, c'est-à-dire les industries reliées à la consommation de masse et de services. D'un point de vue économique, l'exploitation minière, la production de produits ménagers ou de machines outils représentent toutes le même intérêt. Cependant, la spécialisation dans laquelle s'est engagée le Québec comporte certains dangers: à ce titre, il faut avouer que la province est demeurée largement tributaire de l'extérieur pour l'acquisition de la technologie. De plus, la présence de ces firmes engendre des effets sur la balance des paiements, qui ne sont pas sans affecter le Québec. En somme, elles ont eu des effets indirects et induits qui comportent leurs propres limites. À long terme, ce mode de développement comporte le risque que les effets d'entraînement s'estompent jusqu'à être inhibés complètement, dans l'éventualité où le dynamisme de la filiale, comme pôle de croissance, s'effrite. Ce risque est d'autant plus grand que la firme mène une stratégie mondiale de développement, plutôt que nationale. Les intérêts présidant à ses activités ne correspondent pas nécessairement à ceux du Québec, aujourd'hui à la recherche d'une croissance plus diversifiée.

Une étude sur les élites industrielles au Québec fait ressortir que les dirigeants des FMN préferent acheter une unité de production déjà existante dans une proportion de près de $70 \%$, plutôt que de la créer ${ }^{11}$. Ceci relativise certains effets bénéfiques habituellement identifiés à l'arrivée de capitaux étrangers. Les fonctions dévolues à la province en font un lieu d'exécution de leurs plans de production qui n'a pas toujours les retombées économiques souhaitées sur le tissu industriel.

11. Le Groupe de Recherche sur les Élites industrielles au Québec, op. cit., p. 137. 
Une dichotomie se manifeste au chapitre des secteurs d'activités pour lesquels on observe une concentration des avoirs selon l'origine et les secteurs. Mais l'insertion d'une région au sein d'une zone d'intégration ne se fait pas selon des modalités toujours préétablies. La fonction économique n'est pas figée; toutefois, une tendance s'affiche. La force structurante du contexte continental a favorisé le développement du Québec par le biais du secteur primaire, nommément dans le domaine des matières premières et de l'énergie hydro-électrique, ce qui situe grosso modo le rôle de la province dans la division nord-américaine du travail. Aujourd'hui, l'utilisation répandue de l'aluminium comme substitut à l'acier, a permis d'élargir notre base économique. Produit au Québec depuis les années vingt, ce métal y est de plus en plus transformé. De plus, des capitaux américains ont été investis pour la fabrication de matériel de transport, incluant le véhicule privé et les produits aéronautiques et pneumatiques ${ }^{12}$.

La partie des facteurs de production affectée aux activités minières engendre peu de retombées sur la structure industrielle; la technologie utilisée est importée des États-Unis et les métaux sont exportés à la suite d'un processus banal de transformation. Les retombées sur l'emploi et la masse salariale sont importantes, car l'augmentation corollaire de la demande favorise entre autres certains secteurs. Toutefois, l'ensemble économique demeure mal articulé et dépendant d'éléments exogènes fondamentaux.

Pour sa part, le secteur des services a connu une progression au cours des récentes années. C'est dans cette veine que le matériel de transport, l'informatique et les projets de développement hydroélectrique ont connu une forte poussée, simultanément au commerce et à la finance.

12. M. Saint-Germain, op. cit., p. 111 . 
À l'origine, les investissements du secteur primaire ont parfois été motivés par les besoins des centres industriels américains. L'augmentation de la population et du pouvoir d'achat, la diversification et l'augmentation de la production ont entrâné la diversification relative des investissements afin d'exploiter ce nouveau marché ${ }^{13}$.

Cet élargissement s'est surtout réalisé à la faveur du secteur tertiaire qui absorbe par le fait même de plus en plus d'emplois dont le pourcentage atteignait $65 \%$ en $1980^{14}$. La base économique du Québec est demeurée déficiente, étant axée sur la production d'un nombre limité de produits. Certains avantages comparatifs ont été développés, mais ils ont aidé à faire du Québec une société où la consommation de masse a précédé l'élaboration d'un processus productif autocentré et dynamique.

\section{c) Les exportations}

La configuration de nos échanges internationaux, notamment ceux effectués avec les États-Unis, indique les lacunes de notre structure économique. Une caractérisation proposée par Bernard Bonin, nous en indique les grands traits; elle comporte quatre points. Premièrement, un tiers de ces exportations est composé de produits finis et un autre tiers de matières premières. Deuxièmement, elles sont fortement concentrées sur quelques pays de destination; les États-Unis en absorbent plus de $60 \%{ }^{15}$. Troisièmement, plus de $50 \%$ reposent sur quelque dix produits. Quatrièmement, la tendance commerciale du Québec est sensiblement la même que

13. IBID, p. 255 .

14. L'annuaire du Québec, 1979-1980. Québec, l'Éditeur Officiel, 1980, pp. 470471.

15. De $64.4 \%$ qu'il était en 1981 , ce pourcentage a été ramené à $62.7 \%$ en 1982. Secrétariat au Développement Économique du Québec., op. cit., p. 3. 
celle de l'ensemble du Canada; en conséquence, 50 à $60 \%$ de nos exportations doivent vraisemblablement découler du commerce intrafirme ${ }^{16}$. En 1980, la liste des dix produits d'exportation les plus importants comprenait le papier d'imprimerie, les minerais, concentrés et déchets de fer, l'aluminium, les automobiles, et les châssis, le cuivre, les pièces et moteurs d'avions, l'amiante non manufacturée, les métaux précieux, la pâte de bois et les pâtes similaires et le bois d'œuvre. Exception faite de l'automobile et des chassis, du papier, des pièces d'aluminium et des moteurs d'avions, les autres produits appartiennent soit au secteur primaire, ou à celui de la semi-transformation ${ }^{17}$. De plus, $75 \%$ du commerce avec les États-Unis est dirigé vers les États du Nord-Est américain, ce qui rétrécit davantage l'espace de dépendance.

L'intégration du Québec à l'espace nord-américain se fonde principalement sur ses richesses naturelles: l'importance du commerce intrafirme indique quant à lui le rôle important des FMN dans ce processus. Certaines entreprises locales arrivent à percer le marché américain. Toutefois, pour des raisons liées aux réseaux commerciaux et vraisemblablement au mode de gestion de ces entreprises, l'importance relative de leurs exportations est assez faible.

\section{d) La situation de crise}

Toute analyse de la conjoncture économique du Québec doit intégrer la variable nord-américaine, car le Québec subit les variations conjoncturelles de l'économie américaine. Comment pourrait-il en être autrement dans le contexte de dépendance

16. B. Bonin, "USA-Quebec Economic Relations: a background paper", op. cit., p. 3.

17. C. Nappi, op. cit., p. 24. 
structurelle précédemment évoqué? De plus, la fragmentation sectorielle de la base économique du Québec rend aléatoire les tentatives visant à contrer les dérèglements cycliques et/ou structurels provoqués par l'extérieur. La faiblesse de notre secteur manufacturier n'est pas compensée par l'importance du secteur tertiaire ou du secteur primaire. La place accordée à l'exploitation des matières premières dans une stratégie industrielle comporte des limites. L'exploitation de ces matières premières encourt des coûts énormes lorsque la demande pour ces produits chute, ou lorsque les prix tombent. Le dynamisme du processus de développement est alors gravement perturbé. C'est précisément ce qui s'est produit pour les produits de base et au plan de la demande et des prix ${ }^{18}$.

Les Américains se procurent au Québec $80 \%$ de leur amiante, $70 \%$ de l'aluminium importée, $45 \%$ des importations de papier journal, $40 \%$ de celles de fer concentré et un tiers des importations de pièces et de moteurs d'avion, de cuivre et de zinc ${ }^{19}$. Toute fluctuation de leur demande pour ces produits, liée à l'augmentation de la concurrence mondiale, rend le Québec vulnérable aux changements conjoncturels des États-Unis.

Ces perturbations se répercutent sur les infrastructures relevant du secteur privé et du secteur public qui doivent être financées. Ce ralentissement affecte notamment l'industrie du bois de sciage qui fonctionnait à seulement $63 \%$ de sa capacité à la fin de 1982 . Ce pourcentage a atteint $75 \%$ en fin d'année dans l'industrie des pâtes et papier. La situation était encore plus sombre dans le

18. Les exportations de minerais ont accusé une baisse de $17 \%$ au cours des premiers mois de 82; celle-ci était encore plus marquée pour les envois à destination des États-Unis. Seuls le papier d'imprimerie et les produits agro-alimentaires ont enregistré une hausse appréciable. Secrétariat au Développement Économique du Québec, op. cit., pp. 2-5. op. cit..., p. 20 .

19. B. Bonin, «U.S.A. - Quebec Economic Relations; Some Interactions..., 
domaine minier qui maintenait son niveau d'activité à la moitié de sa capacité pendant l'été 1982 , ce qui a occasionné la mise à pied de $40 \%$ des effectifs ${ }^{20}$. Le financement de méga-projets à des taux d'intérêts élevés est très onéreux. De plus, ce mode de développement favorise peu les petites unités locales de production, qui se tiennent à l'écart des réseaux d'exportation. La politique économique d'un pays ne doit pas traiter exclusivement des questions de rentabilité. Les problèmes de l'emploi, des disparités régionales et d'égalité des chances doivent être intégrés. Le comportement économique de la firme peut entrer en contradiction avec ces considérations sociales. Son origine extraterritoriale et son orientation productiviste sont de nature à aviver certains conflits sociaux dans le pays d'accueil. Cette orientation est encore plus marquée chez les FMN dont le coût relatif de déplacement hors d'un pays est moins élevé que pour une entreprise d'envergure nationale. Le déplacement d'unités de production provoque des perturbations au sein des collectivités touchées par le chômage. De plus, la place importante que les FMN occupent dans notre économie, exerce un puissant impact sur la répartition sectorielle de l'emploi.

La force avec laquelle la crise actuelle frappe le Québec est un indice de la fragilité de la structure productive et elle fait ressortir certains dangers à entretenir des relations économiques inévitablement trop exclusives avec un seul pays. Les États-Unis qui détenaient $21 \%$ du commerce mondial en 1970, n'en contrôlaient plus que $16 \%$ en 1983 . De plus, le Québec entretient la majeure partie de ses relations économiques avec le Nord-Est américain. Or, les pôles de développement économique aux ÉtatsUnis se déplacent du Nord-Est vers le Sud-Ouest. Vu leur stagnation

20. Secrétariat au Développement Économique du Québec, op. cit., p. 8. 
relative, ces régions sont peu susceptibles de dynamiser davantage notre économie.

La crise actuelle exacerbe les coûts imputables à la présence du capital multinational. En plus de devoir payer des dividendes et autres redevances, la présence étrangère peut conduire à l'inefficacité et à la limitation de l'exercice de la souveraineté nationale. B. Bonin a identifié quelques éléments constituant des limites de l'investissement étranger. La tendance à l'augmentation de la concentration de l'activité économique, les pratiques restrictives en matière de commerce international et les distorsions apparaissant dans le processus de développement économique, seraient autant d'exemples d'inefficacité. Sur le plan politique, les choix de gouvernements sont réduits dans la mesure où les firmes peuvent user de représailles ${ }^{21}$.

Certains enjeux réels apparaissent de façon plus claire, en l'occurrence le contrôle du système économique, incluant les réseaux de production et de distribution.

\section{Nationalisme ou continentalisme}

Au cours de son histoire, le Québec a eu à prendre position sur des questions concernant les États-Unis. Le gouvernement central du Canada a posé plusieurs choix au nom du pays tout entier. Néanmoins, la question du renforcement de nos relations avec les États-Unis a été soulevée à plusieurs reprises depuis que les treize États de la Nouvelle-Angleterre ont rompu leurs liens coloniaux. L'hésitation à nous engager au resserrement des liens ou au maintien d'une distanciation, relève vraisemblablement des

21. B. Bonin, «U.S.A. - Quebec Economic Relations; Some Interactions..., " op. cit., p. 27. 
paradoxes sous-jacents à l'une ou l'autre des options ${ }^{22}$. Ces choix politiques concernaient le type de stratégie économique nationale. La médiation des institutions politiques a donc joué, et joue toujours, un grand rôle dans l'insertion d'un pays dans les rapports économiques internationaux.

D'un point de vue économique, l'attrait du savoir-faire et du marché constitue une force d'attraction. Par contre, le désir de construire des avantages comparatifs dans des secteurs autres que ceux privilégiés par les Américains induit un rôle différent aux agents internes et externes. L'éternel débat autour du continentalisme a été relancé en janvier 1983 par une déclaration du ministre Landry en faveur de l'établissement d'un marché commun entre le Québec et les États-Unis ${ }^{23}$. Cette option peut se motiver par le désir d'abaisser le coût des produits importés des ÉtatsUnis, d'intégrer davantage les processus productifs, ce qui entraînerait une intégration plus poussée des marchés. Il ne faut toutefois pas perdre de vue les visées politiques sous-jacentes à cette démarche. Malgré les avantages de cette approche, il faut évoquer ses dangers politiques.

Comme le mentionnait Paul Painchaud dans un article portant sur nos relations avec les États-Unis, «il n'y a pas d'économie internationale pure: tout est politique dès lors qu'il s'agit de relations avec l'étranger ${ }^{24}$.

22. Au sujet de cette ambivalence, le lecteur peut consulter le texte de $\mathbf{L}$. Balthazar, "Les relations canado-américaines", dans Le Canada et le Québec sur la scène internationale, édité par Paul Painchaud. Québec, PUQ, 1977, pp. 308-328.

23. L'article suivant reproduit l'essentiel de la déclaration du ministre Landry: "Le Québec et le Canada auraient tout à gagner à établir un marché commun avec les États-Unis", dans Le Devoir, LXXIV, 18, lundi, 24 janvier 1983, p. 2.

24. P. Painchaud, "La Sudpolitik: le devoir de discipline», dans Le Devoir, LXXIV, 38 mercredi, 16 février 1983, p. 9. 
Le choix posé par nos dirigeants politiques comporte des aspects à la fois positifs et négatifs. L'intégration continentale pourrait faciliter l'approfondissement d'un vaste marché. D'une part, elle rendrait le Québec plus dépendant de pôles de décision. Déjà, la présence des FMN circonscrit en partie le rôle économique laissé aux entreprises locales dont la participation à la vie économique semble délimitée par l'espace non occupé par les grandes firmes. Et pourtant, les autorités politiques actuelles disent miser sur les PME. La politique économique d'un Québec inféodé davantage aux États-Unis serait plus sujette aux pressions politiques et économiques américaines. L'écart entre les deux gouvernements en matière économique laisse déjà peu de marge de manœuvre au Québec dans ses choix économiques. L'intégration risquerait de les réduire encore. L'approfondissement de cet espace nord-américain impose des limites aux initiatives de développement régional.

D'autre part, le Québec a besoin de capitaux pour se développer, tout autant que d'un marché extérieur pour écouler sa production. Les relations politiques conditionnées par la proximité géographique, soit la géopolitique, et l'importance actuelle des États-Unis comme partenaire commercial du Québec incitent à la prudence. Cela ne signifie pas que nos dirigeants doivent suivre la voie d'un réalisme politique invétéré qui condamne le Québec à ramasser ce que les Américains veulent bien lui accorder. Malgré les limites imposées par la géopolitique, les dirigeants disposent d'une autonomie relative dans les choix qu'ils posent.

La politique de rapprochement des pays, à l'intérieur de rassemblements plus ou moins imperméables, c'est-à-dire les mouvements d'intégration régionale, incite la province à protéger ses arrières en se rangeant dans un camp. Cependant les ÉtatsUnis ne connaissent plus la croissance économique d'antan: leur rôle stimulateur peut s'avérer mitigé.

La tendance bien ancrée des FMN à favoriser le commerce intrafirme ne sera pas radiéee avec la continentalisation accélérée. 
De plus, aucune mécanique n'assure que la base économique du Québec serait plus diversifiée par un processus accélérée d'intégration. D'ailleurs, l'expérience européenne indique que l'intégration économique accroît la spécialisation des économies. La diversification de l'économie québécoise risquerait donc d'être ralentie par l'intégration nord-américaine qui favoriserait les secteurs dans lesquels le Québec a déjà acquis des avantages comparés. Le Québec peut choisir d'acquérir ces avantages par le truchement de la FMN ou il peut opter pour un interventionnisme ou toute autre forme d'organisation économique impliquant des acteurs locaux. La réalité politique et économique conditionne la prise de décision, mais le réalisme ne doit pas couper les dirigeants des projets susceptibles de consolider la base économique du Québec. Les tentatives de formulation d'une politique industrielle cohérente pour le Québec avec la publication de Bâtir le Québec (1979) et Le Virage technologique (1982) indiquent les problèmes de diversification de la base économique. Or, les débats actuels sont suffisamment importants pour indiquer les différences d'intérêts liées aux divergences de vues. La mise sur pied d'un marché commun constitue un choix politique dont il convient de saisir toutes les dimensions.

\section{Conclusion}

La région a acquis un caractère périphérique que lui confere son inégalité structurelle. Toute politique de reprise en main de l'économie peut nous entraîner dans une situation de déséquilibre important. Mais celle prévalant aujourd'hui n'est pas étrangère au chômage, à la réduction de la productivité ou à la déflation économique. L'intériorisation élargie des relations actuelles du Québec avec notre puissant voisin est une question éminemment politique. Tout dépendra des choix politiques du Gouvernement. Malgré les influences externes multiples, la responsabilité de la 
décision revient aux hommes politiques qui doivent choisir entre une spécialisation nord-américaine par une rationalisation des activités, le maintien d'une relative indépendance économique destinée à orienter le développement économique selon une politique industrielle nationale, ou tout autre projet conciliant les soucis d'indépendance et d'efficacité. L'un ou l'autre de ces choix n'élimine pas les Américains des circuits économiques québécois. Cependant, la pondération des rôles respectifs serait différente, de même que la possibilité pour le Québec de contrôler son devenir. En avril 1972, le Président Nixon présentait à Ottawa ce qui est devenu la base des relations politiques bilatérales. La Doctrine Nixon rappelait que le Canada et les États-Unis sont deux entités spécifiques aux intérêts particuliers et que les politiques de chacun doivent être autonomes et indépendantes ${ }^{25}$. L'étendue de la coopéraiton actuelle ne doit pas faire oublier le caractère spécifique des intérêts. Les politiques indépendantes et autonomes souhaitées par le Président Nixon s'accommodent mal d'une dépendance économique qui traduit une inégalité structurelle importante. Aucune logique mécanique n'a orienté la politique continentale jusqu'à maintenant; elle a été le résultat de rapports de forces économiques et de décisions politiques. Il ne semble pas y avoir davantage de relations strictement naturelles que de «gros bon sens» en politique. Tout dépend des objectifs fixés par l'État. Paradoxalement, certains nationalistes québécois souhaitent que les relations avec les ÉtatsUnis s'approfondissent pour faire contrepoids à l'influence canadienne-anglaise ${ }^{26}$. Besoin est de s'interroger sur les dangers que ce contrepoids ne devienne à son tour contraignant à tous égards.

25. J. W. Holmes, "Impact of Domestic Political Factors on Canadian American Relations: Canada", dans Canada and the United States; Transnational and Transgovernmental Relations, édité par A. B. Fox, A. O. Hero et J. S. Nye. New York, Columbia University Press, 1976, pp. 19-20.

26. L. Bissonnette "The Evolution of Quebec-American Diplomacy", dans Contemporary Quebec, édité par C. Veltman. Montréal, UQAM, 1981, p. 167. 
Faut-il rappeler que la sympathie américaine vis-à-vis du mouvement national québécois n'est pas particulièrement virulente? Au contraire! Et réciproquement, une partie très importante de l'élite politique et économique régionale souhaite approfondir les relations avec les Américains dans la perspective à court terme de meilleures affaires. Ces intérêts fractionnels ne peuvent pas être considérés comme garants des intérêts à long terme plus vastes. Il n'est pas rare de les voir se soulever contre des politiques visant à accroître le contrôle national sur un secteur d'activité. L'engagement des provinces dans le domaine des relations internationales reflète des intérêts internes. Cela dit, comment une plus grande intégration aux États-Unis garantirait-elle le respect de cette spécificité? La force relative du Québec par rapport aux États-Unis est moins grande que par rapport au reste du Canada. Comment peut-on alléguer que le Québec serait plus à même de renégocier les modalités d'une intégration nord-américaine alors qu'il arrive difficilement à faire prévaloir ses vues au sein du Canada?

Les relations américano-québécoises englobent un champ de dépendance et de déterminations dont le Québec est la partie faible. La force économique est à même de conduire à l'imposition de modalités économiques. Cette détermination extérieure plaide en faveur d'une diversification des partenaires du Québec. Cependant, la difficulté pour un pays de s'introduire à l'intérieur d'un regroupement économique oblige à nuancer l'analyse. On peut s'interroger sur les possibilités réelles de la province de développer de nouveaux avantages comparatifs avec le Japon ou la CEE qui sont des centres économiques constitués. Une étude réalisée en 1980 évaluait à $7.8 \%$ le potentiel de substitution des importations américaines par des importations européennes ou japonaises $^{27}$. De plus, les apports extérieurs qu'ils exigent sont

27. J.-E. Denis et E. Lindekens "Third Option never Given a Fair Chance», dans The Canadian Business Revieu', vol. VII, n"1, printemps 1980. p. 6. 
dans le secteur des matières premières et dans celui de la technologie hautement sophistiquée. Dans le premier cas, le Québec maintiendrait une inégalité structurelle jusqu'ici dénoncée. Dans le second, il est pratiquement absent. Les secteurs mitoyens intéressants pour le Québec existent assurément, mais il faut reconnaître qu'ils sont à identifier et à développer.

Les solutions aux problèmes reliés aux relations Québec/ États-Unis comportent leurs ambiguittés et leurs zones d'incertitudes. D'un point de vue strictement commercial, les échanges mondiaux sont encadrés par l'interventionnisme des États lié à certaines politiques restrictives des firmes elles-mêmes. Le rééquilibrage de nos relations commerciales se heurte immanquablement à des limites structurelles. Mais une telle constatation ne fait que situer la force d'attraction économique qui retient le Québec au géant américain. Mais cela n'empêche pas d'explorer toutes les possibilités. À ce chapitre, il ne faut pas négliger les pressions exercées par la population sur le gouvernement. Les pouvoirs publics doivent considérer les tendances majeures de l'opinion publique avant d'adopter les moyens à mettre en œuvre. Cependant, l'illusion joue aussi à ce niveau. La socialisation et l'acculturation constituent une force d'attraction qui prédispose à un certain choix. D'où l'importance du culturel.

Somme toute, l'arrière-plan politique et économique des relations Québec/États-Unis indique les limites structurelles avec lesquelles les dirigeants du Québec doivent composer. Il ne faut pas tomber dans une vision catastrophique décrivant des dirigeants aux pieds et mains liés par des liens de dépendance absolue qui les unissent aux centres de décisions américains. À l'autre extrême, l'illusion de la souveraineté des États où les décisions seraient le fruit de choix strictement internes ne doit pas non plus être cultivée. La réalité se situe quelque part entre ces deux extrêmes. Les relations politiques et économiques internationales s'expriment 
à l'intérieur d'une hiérarchie des États émanant de la force relative de chacun d'entre eux. Un État dépourvu de fondements économiques substantiels pourra difficilement contrer les pressions exercées par des partenaires économiques plus puissants. Un autre pays plus fort pourra manifester davantage d'automonie et déterminer les règles du jeu à l'intérieur, voire à l'extérieur, de son territoire.

Le concept d'espace est utile pour saisir la configuration de cette hiérarchie. L'espace définit à la fois les enjeux et les forces structurantes des instances sociales, politique et économique. Les relations du Québec avec les États-Unis s'expriment conformément à cette hiérarchie, toujours mouvante, caractérisée par une intégration structurelle relativement élevée des tissus économiques nationaux.

L'État Québécois peut toutefois se prévaloir d'une relative autonomie comme de fut le cas notamment lors de la création des Sociétés d'État, Hydro-Québec, la Caisse de dépôt et de placement, la Société générale de financement, Sidbec et autres, dont l'importance économique est aujourd'hui considérable. Ces initiatives étatiques non négligeables ont d'ailleurs pavé le chemin à la constitution d'une bourgeoisie québécoise liée à ces institutions, venue s'ajouter à la petite bourgeoisie dont l'existence est associée aux PME.

La volonté du gouvernement du Québec de diversifier ses partenaires économiques n'a peut-être pas porté tous les fruits escomptés; toutefois, l'arrivée de Pechiney illustre une réussite allant dans ce sens. Mais celles-ci sont tout de même très limitées.

La forte proportion du PNB québécois reposant sur les exportations $(30 \%)$ de même que l'importance du marché américain pour écouler ces produits indiquent une certaine vulnérabilité de l'économie provinciale par rapport à l'économie américaine. Au plan politique, la susceptibilité américaine entourant la question 
nationale liée au problème constitutionnel des responsabilités respectives des deux paliers de gouvernement en matière de relations extérieures placent les dirigeants québécois dans une situation délicate, rendant un peu plus ardue la formulation d'une politique internationale pour le Québec intégrant de façon cohérente les États-Unis. Il ne faut pas se faire bercer par l'illusion légale de la souveraineté des Étas car ni Ottawa ni Québec ne peuvent faire abstraction de leur puissant voisin lors de l'adoption d'orientations politiques. L'espace semble être un concept utile pour appréhender le dynamisme entourant les relations du Québec avec les ÉtatsUnis. 